\title{
Acceptance situation of HIV patients in Japanese dialysis facilities-questionnaire survey by the Infection Survey Subcommittee
}

\author{
Ayumi Yoshifuji ${ }^{1}$, Munekazu Ryuzaki ${ }^{1} 2^{*}$, Yasuhiko Ito ${ }^{1}$, Norio Ohmagari ${ }^{1}$, Yoshihiko Kanno ${ }^{1}$, Toshio Shinoda', \\ Yaoko Takano', Isao Tsukamoto', Kazuhiko Hora', Yasushi Nakazawa', Naoki Hasegawa', Tadashi Yoshida', \\ Shu Wakino ${ }^{1}$, Yoshiaki Takemoto ${ }^{1}$ and Hidetomo Nakamoto ${ }^{1}$
}

\begin{abstract}
Background: A newspaper article in August 2016 reported that about 40 medical facilities refused dialysis to a human immunodeficiency virus (HIV) patient. Infection Survey Subcommittee in the Japanese Society for Dialysis Therapy investigated this situation.

Methods: An anonymous questionnaire survey about the acceptance of HIV-positive patients was sent to 4039 dialysis institutions. The questionnaire included a number of HIV-positive patients on dialysis currently and in the last 5 years, details on whether the institution received requests to perform dialysis on HIV-positive patients, the responses to these requests, the preparation system for acceptance, the reasons for refusal of dialysis of HIV-positive patients, knowledge of HIV-related guidelines in use, and the considerations for the acceptance of dialysis for HIV-positive patients. We evaluated answers to these questions from the institutions.

Results: Two thousand five hundred eighty-three facilities (64.0\%) responded to the survey questions. In the past 5 years, 215 facilities (8.3\%) were requested to accept HIV patients for dialysis and $40.1 \%$ of these refused acceptance. Although manuals, written policy and procedure, for a needle piercing accident existed in most of the facilities that accepted the patients, many facilities did neither prepare the stocks of anti-HIV drugs nor cooperate with HIV core hospitals. Principal reasons for the refusal were listed as the lack of medical experience with HIV patients, anxiety of healthcare providers with regard to HIV infection risk, lack of stocks of anti-HIV drugs for needle piercing accidents, and the lack of cooperation with HIV core hospitals. The proportion of facilities expected to accept HIV patients in the future was as low as $16.9 \%$ among all facilities that responded to the survey.
\end{abstract}

Conclusions: The Japanese Society for Dialysis Therapy will need to strengthen the cooperation with other academic societies and disseminate basic knowledge on HIV and infection control measures to develop and establish the acceptance system of maintenance dialysis for HIV patients.

Keywords: HIV, Maintenance dialysis, Acceptance, Refusal, Infection control

\footnotetext{
* Correspondence: ryuzaki-m@saichu.jp

${ }^{1}$ Infection Survey Subcommittee, Japanese Society for Dialysis Therapy,

Tokyo, Japan

${ }^{2}$ Division of Nephrology, Department of Internal Medicine, Tokyo Saiseikai

Central Hospital, 1-4-17 Mita, Minato-ku, Tokyo 108-0073, Japan
}

(c) Japanese Society for Dialysis Therapy. 2018 Open Access This article is distributed under the terms of the Creative Commons Attribution 4.0 International License (http://creativecommons.org/licenses/by/4.0/), which permits unrestricted use, distribution, and reproduction in any medium, provided you give appropriate credit to the original author(s) and the source, provide a link to the Creative Commons license, and indicate if changes were made. The Creative Commons Public Domain Dedication waiver (http://creativecommons.org/publicdomain/zero/1.0/) applies to the data made available in this article, unless otherwise stated. 


\section{Background}

The AIDS Surveillance Committee of Ministry of Health, Labour and Welfare in Japan reported that the cumulative number of patients infected with human immunodeficiency virus (HIV) was 27,443 in 2016, which is increasing with new registrations of approximately 1500 patients every year [1]. Due to the progress of antiviral agents, HIV-positive patients today are expected to have nearly the same life prognosis as that of non-HIV patients. The consideration of HIV infection has changed from "a deadly illness" to "a treatable chronic disease" in recent years. Therefore, various chronic diseases associated with HIV infection have drawn attention. Chronic kidney disease in HIV patients, also known as HIV-CKD, is also considered one of these examples. Though the proportion of patients requiring the maintenance dialysis is still unknown, it is reported to be $0.5-1.0 \%$ in the Western countries $[2,3]$. The increase in number of HIV-CKD patients is predicted to result in an increase in number of patients requiring maintenance dialysis in near future. The shocking article from the "Tokyo Shimbun" on 30th of August in 2016 reported that a HIV-positive patient requiring hemodialysis was refused maintenance dialysis by about 40 medical institutions.

Therefore, the Infection Survey Subcommittee in the Japanese Society for Dialysis Therapy conducted a questionnaire survey for secure healthcare access at the member institutions.

\section{Methods}

An anonymous questionnaire survey about the acceptance of HIV-positive patients was sent to 4039 dialysis institutions including hospitals and clinics, which were registered as member institutions of the Japanese Society for Dialysis Therapy in November 2016.

The questionnaire included the location, type, and number of dialysis patients as well as number of HIV-positive patients on dialysis currently and in the last 5 years at an institution as of 1st of November in 2016. Details on whether the institution received requests to perform dialysis on HIV-positive patients, the responses to these requests, the preparation system for acceptance (manuals, written policy and procedure, for a needle piercing accident, cooperation with HIV core hospitals, preparation of the stocks of anti-HIV drugs, and the payments for these drugs), the rate of needle piercing accidents at the accepting institution, the reasons for refusal of dialysis of individual HIV-positive patients, the general reasons to refuse dialysis of HIV-positive patients, knowledge of HIV-related guidelines in use ("the Guidelines for Dialysis of HIV Positive Patients" published by Japanese Association of Dialysis Physicians and Japanese Society for Dialysis Therapy in 2010, "the Guidelines for Fundamental Handling and Infection
Control in Dialysis Facilities in the fourth revised edition" published in 2015, and "Treatment guidelines for HIV" published by the research division of Ministry of Health, Labour and Welfare in 2016), the considerations for the acceptance of dialysis for HIV-positive patients, and the pros and cons of the transfer of results of this questionnaire to the HIV core hospitals.

\section{Results}

The anonymous questionnaire was sent to 4039 dialysis institutions registered as member institutions of the Japanese Society for Dialysis therapy, and 2583 facilities (64.0\%) answered the questionnaire. With respect to the types of institutions, hospitals accounted for $47.7 \%$ (1232 facilities), hemodialysis clinics accounted for 35.1\% (907 facilities), and university hospitals accounted for 3.8\% (98 facilities). The locations of the facilities and the number of patients in each are shown in Fig. 1.

The number of HIV-positive patients currently on dialysis in these facilities was 87 (Table 1). The number of HIV-positive patients on dialysis in the past 5 years was 228 (Table 2). The facilities which received requests to accept dialysis for HIV-positive patients among member institutions in the past 5 years accounted for 8.3\% (215 facilities). $40.1 \%$ of these refused acceptance although $52.5 \%$ of these accepted (Table 3).

When facilities accept HIV-positive patients, it is recommended to prepare a manual for needle piercing accidents, cooperation with HIV core hospitals, and the stocks of anti-HIV drugs. $96.6 \%$ (200 facilities) of the accepting facilities prepared the manual for a needle piercing accident. However, among these, only 59.6\% (124 facilities) directly cooperated with HIV core hospitals and only $51.1 \%$ (121 facilities) prepared the stocks of anti-HIV drugs for a needle piercing accident (Table 4). $78.8 \%$ (93 facilities) with the stocks of anti-HIV drugs pay their expenses by themselves, 7.6\% (9 facilities) request the expenses to be paid by the HIV core hospitals, and $2.5 \%$ request the expense to be paid by the staff. In fact, a needle piercing accident occurred in $12.9 \%$ (27 facilities) of these facilities. Although $96.3 \%$ of these managed the accident as per the existing manual, 3.7\% ( 1 facility) experienced difficulties to deal with the accident due to panic among healthcare providers. With respect to the time to administer the anti-HIV drugs after the accident, the proportion of facilities "within $3 \mathrm{~h}$ " accounted for $66.7 \%$ (14 facilities), followed by "within $6 \mathrm{~h}-9.5 \%$ ( 2 facilities), and "within $12 \mathrm{~h}$ " $-14.3 \%$ ( $3 \mathrm{fa}-$ cilities). This implies that every person in each facility received the preventive medicine within $12 \mathrm{~h}$, except for personnel in facilities $(9.5 \% ; 2$ facilities) which did not select the preventive medicine. With respect to the time to the consultation with a HIV specialist in the respective HIV core hospital, $71.4 \%$ (10 facilities) consulted 


\section{The general description of dialysis facilities that responded to the questionnaire survey}

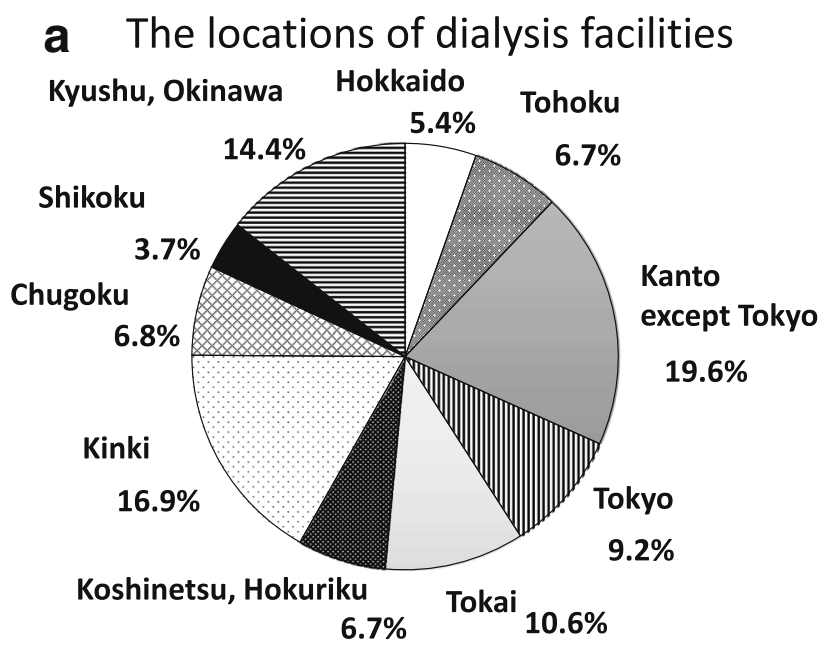

The number of valid responses 2,582

b The number of dialysis patients in these facilities

\begin{tabular}{|l|c|r|}
\hline $\begin{array}{l}\text { the number } \\
\text { of patients }\end{array}$ & $\begin{array}{l}\text { the number of } \\
\text { responses }\end{array}$ & \multicolumn{1}{|c|}{$\%$} \\
\hline $1-9$ & 86 & $3.3 \%$ \\
\hline $10-49$ & 772 & $29.9 \%$ \\
\hline $50-99$ & 944 & $36.5 \%$ \\
\hline $100-199$ & 595 & $23.0 \%$ \\
\hline $200-299$ & 82 & $3.2 \%$ \\
\hline $300-399$ & 25 & $1.0 \%$ \\
\hline $400-499$ & 5 & $0.2 \%$ \\
\hline $500-$ & 7 & $0.3 \%$ \\
\hline No response & 67 & $2.6 \%$ \\
\hline
\end{tabular}

Fig. 1 The general description of dialysis facilities that responded to the questionnaire survey. The regions of dialysis facilities that responded to the questionnaire survey are shown in Fig. 1a. We divided them into 10 regions from north to south, i.e., Hokkaido, Tohoku, Kanto except Tokyo, Tokyo, Tokai, Koshinetsu/Hokuriku, Kinki, Chugoku, Shikoku, and Kyushu/Okinawa. We received similar rate of responses from each region. The number of dialysis patients in these facilities is shown in Fig. 1b

immediately, $21.4 \%$ (3 facilities) on the next day, and $7.1 \%$ (1 facility) after 2 days (Fig. 2).

About $40.1 \%$ of the facilities refused acceptance of HIV-positive patients due to the following reasons: no experience in the practice of HIV-positive patients, anxiety among the healthcare providers with respect to HIV infection risk, lack of anti-HIV drug stocks for a needle piercing accident, lack of cooperation with the HIV core hospitals, insufficient standard precautions in medical facilities, lack of knowledge on the required measures during a needle piercing accident, and insufficient beds for dialysis (Table 5). Moreover, the general reasons for refusing healthcare to HIV-positive patients, which were evaluated at all facilities, were similar to those listed above (Table 6).

With respect to cooperation with facilities that have experiences accepting HIV-positive patients or with HIV core hospitals, only $8.9 \%$ (221) of the facilities have cooperated in the past and $17.9 \%$ (443 facilities) plan to cooperate in future (Table 7).

Three guidelines are known for performing dialysis of HIV-positive patients, which include "the Guidelines for Dialysis of HIV Positive Patients" published by Japanese Association of Dialysis Physicians and Japanese Society 
Table 1 The number of HIV positive patients on dialysis in a facility

\begin{tabular}{|c|c|c|}
\hline (No. of persons & The number of responses & $\%$ \\
\hline c & 2483 & $96.1 \%$ \\
\hline & 58 & $2.2 \%$ \\
\hline 2 & 13 & $0.5 \%$ \\
\hline 3 & 1 & $0.0 \%$ \\
\hline No answer & 28 & $1.1 \%$ \\
\hline
\end{tabular}

for Dialysis Therapy in 2010, "the Guidelines on Fundamental Handling and Infection Control in Dialysis Facilities in the fourth revised edition" published in 2015, and "Treatment Guidelines for HIV" published by the Research Division of Ministry of Health, Labour and Welfare in 2016 [4-6]. The actual compliance of these guidelines was really low based on the high frequencies of facilities unfamiliar, vaguely familiar, or familiar without practice of these guidelines, as shown in Table 8 . The proportion of the facilities that never knew about all these guidelines accounted for 3.8\% (98 facilities).

The proportion of facilities intending to accept HIV-positive patients for dialysis in future was as low as $16.9 \%$ (436 facilities), while the proportion of facilities intending to refuse it was $38.3 \%$ (988 facilities). Furthermore, the proportion of facilities selecting "others" accounted for $43.1 \%$ (1114 facilities) (Fig. 3). 20.4\% of the facilities with the intention to accept refused to transfer the results of our questionnaire survey to HIV core hospitals (Fig. 4). Considering the opinions from all facilities, $65.9 \%$ (460 facilities) accepted to transfer the results of this questionnaire survey and $26.1 \%$ refused (data not shown).

\section{Discussion}

The current questionnaire survey, performed in response to the shocking article published in the "Tokyo Shimbun" in 2016 reporting that maintenance hemodialysis in a HIV-positive patient was refused by a series of 40 medical institutions, demonstrated that $40.1 \%$ of the dialysis facilities refuse dialysis for HIV-positive patients and 38.3\%

Table 2 The number of HIV positive patients on dialysis in a facility in the past five years

\begin{tabular}{|c|c|c|}
\hline (No. of persons) & The number of responses & $\%$ \\
\hline c & 2404 & $93.1 \%$ \\
\hline 1 & 107 & $4.1 \%$ \\
\hline 2 & 32 & $1.2 \%$ \\
\hline 3 & 5 & $0.2 \%$ \\
\hline 4 & 3 & $0.1 \%$ \\
\hline More than 4 & 6 & $0.2 \%$ \\
\hline No response & 26 & $1.0 \%$ \\
\hline
\end{tabular}

Table 3 Responses of the request to accept the HIV positive patients

\begin{tabular}{ccc}
\hline & The number of responses & $\%$ \\
\hline Accepted & 114 & $52.5 \%$ \\
$\begin{array}{c}\text { Tried to accept, but } \\
\text { refused by a patient }\end{array}$ & 17 & $7.8 \%$ \\
$\quad$ Refused & 87 & $40.1 \%$ \\
$\quad$ number of & 217 & - \\
valid responses & & \\
\hline
\end{tabular}

would not intend to accept them in future. This shocking result closely matches with the report by Yanagisawa et al. showing that $55.3 \%$ of the facilities will not be willing to accept HIV-positive patients in future [3].

In the past, HIV infection caused patient death within a few years unless they were treated with the appropriate anti-HIV drugs. Multiple anti-HIV drugs have been developed and applied in the clinical practice since the latter half of 1990s. Subsequently, the prognosis of the HIV-positive patients dramatically improved to nearly the same as non-HIV patients. Along with improvements in life prognosis, we need to not only control the AIDS defining illnesses and malignant tumors, that are the principle causes of death in HIV patients, but also the comorbid chronic diseases such as diabetes mellitus, dyslipidemia, chronic kidney disease, and cardiovascular disease [2]. It has been reported that the prevalence of chronic kidney disease in HIV-positive patients in Japan is $15.4 \%$ [7]. Hara et al. reported that chronic kidney disease in HIV-positive patients progresses more rapidly than that in non-HIV patients [8]. Rasch et al. reported that the introduction rate of hemodialysis in HIV positive patients is 3.6 times higher than that of non-HIV patients [9]. Yanagisawa et al. reported in 2014 that the frequency of HIV-positive patients among those treated with hemodialysis is as low as $0.024 \%$ in Japan, which is significantly lower than the frequencies of $1 \%$ in the USA and $0.67 \%$ in France [3]. Our data from the survey estimated that the proportion of HIV-positive patients treated with dialysis (87) among all dialysis patients treated in the facilities that responded to the questionnaire $(217,429)$ was as low as $0.04 \%$. Consequently, these

Table 4 The situation at facilities with respect to preparation for accepting HIV positive patients

\begin{tabular}{lll}
\hline & Yes & No \\
\hline A manual for needle piercing accidents ${ }^{1}$ & $96.6 \%$ & $3.4 \%$ \\
Cooperation with HIV core hospitals ${ }^{2}$ & $59.6 \%$ & $40.4 \%$ \\
Stocks of anti-HIV drugs ${ }^{3}$ & $51.1 \%{ }^{\mathrm{a}}$ & $48.9 \%$ \\
\hline${ }^{\mathrm{a}} 78.8 \%$ of the facilities pay their expenses themselves & & \\
${ }^{1}:$ number of valid responses 207 & \\
${ }^{2}:$ number of valid responses 208 & & \\
${ }^{3}:$ number of valid responses237 & &
\end{tabular}


The measures for a needle piercing accident

\section{Reaction according to the manual}

Create panic

$3.7 \%$

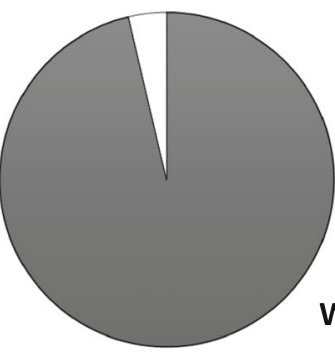

Can react according to the manual

$96.3 \%$

\section{The time to obtain the anti-HIV drugs}

No medicine

$9.5 \%$

Within $12 \mathrm{hrs}$

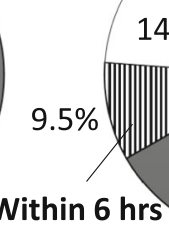

$14.3 \%$

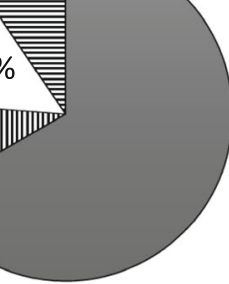

Within 3 hrs

$66.7 \%$

\section{The time to visit the HIV specialists}

More than one day

$7.1 \%$

The next day

$21.4 \%$

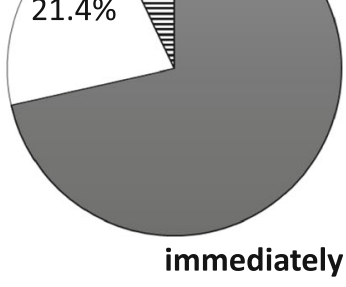

$71.4 \%$

\section{Number of valid responses}

\section{Facilities}

Fig. 2 The measures for a needle piercing accident. A needle piercing accident occurred in 12.9\% of the facilities accepting HIV-positive patients (27 facilities). We evaluated their reactions in the following aspects (1) whether they responded according to the manual, (2) the time duration it took the relevant person to obtain the preventative medicine after the needle piercing accident, and (3) the duration it took for them to consult the HIV specialists

fewer number of HIV-positive patients among all dialysis patients compared to Western countries could cause a delay in the treatment plan for these patients in Japan because of the lack of interest and awareness.

As shown in this study, the principle reasons for the refusing healthcare to HIV-positive patients were the lack of basic knowledge on HIV infection among healthcare providers including insufficient experience of

Table $\mathbf{5}$ The reasons for refusal at facilities that requested to accept

\begin{tabular}{lrr}
\hline & $\begin{array}{c}\text { number } \\
\text { of responses }\end{array}$ & $\%$ \\
\hline Not enough beds & 19 & $17.4 \%$ \\
No experience of the practice & 62 & $56.9 \%$ \\
No acceptance of outpatients & 6 & $5.5 \%$ \\
No cooperation with HIV specialists & 9 & $8.3 \%$ \\
(HIV core hospitals) & & \\
No cooperation with HIV core hospitals & 43 & $39.4 \%$ \\
Insufficient standard precautions in the & 39 & $35.8 \%$ \\
facility & & \\
No knowledge of needle piercing accidents & 19 & $17.4 \%$ \\
No stocks of anti HIV drugs & 57 & $52.3 \%$ \\
Anxiety of HIV infection risk & 60 & $55.0 \%$ \\
Others & 26 & $23.9 \%$ \\
Number of valid responses & 109 & - \\
\hline
\end{tabular}

doctors with HIV-infected individuals, leading to a biased impression, such as the fear of transmitting HIV infection. Our results suggest that the basic knowledge on infection control and prevention of HIV infection are not fully understood in these facilities. These include lower infection rates of HIV than HBV and HCV, (e.g., $0.09 \%$ infection rate after hematic mucosal exposure) and a low infectiousness of HIV-positive patients under

Table 6 The general reasons for refusal in all facilities

\begin{tabular}{lrr}
\hline & $\begin{array}{c}\text { number } \\
\text { of responses }\end{array}$ & $\%$ \\
\hline Not enough beds & 362 & $14.4 \%$ \\
No experience of the practice & 1915 & $76.3 \%$ \\
No acceptance of outpatients & 148 & $5.9 \%$ \\
No cooperation with HIV specialists & 305 & $12.2 \%$ \\
(HIV core hospitals) & & \\
No cooperation with HIV core hospitals & 1130 & $45.0 \%$ \\
Insufficient standard precautions in the facility & 645 & $25.7 \%$ \\
No knowledge of needle piercing accidents & 730 & $29.1 \%$ \\
No stocks of anti HIV drugs & 1280 & $51.0 \%$ \\
Anxiety of HIV infection risk & 1630 & $63.9 \%$ \\
Others & 176 & $7.0 \%$ \\
Number of valid responses & 2510 & - \\
\hline
\end{tabular}


Table 7 Cooperation with facilities that have experiences of accepting HIV positive patients, or with HIV core hospitals

\begin{tabular}{lrr}
\hline & number of responses & \multicolumn{1}{c}{$\%$} \\
\hline Experience of cooperation & 221 & $8.9 \%$ \\
No experience of cooperation & 1612 & $65.2 \%$ \\
Intention of cooperation & 443 & $17.9 \%$ \\
No intention of cooperation & 651 & $26.3 \%$ \\
Others & 140 & $5.7 \%$ \\
number of valid responses & 2472 & -
\end{tabular}

good control with regular antiviral treatment [10]. In addition, appropriate measures after a needle piercing accident are not recognized in these facilities. Infection control for the HIV-infected, as per "the guidelines on fundamental handling and infection control in dialysis facilities in the fourth revised edition," does not ask for patient isolation or fixed position of beds, but only for standard precautions such as hand hygiene and individual protection for healthcare providers [5]. Moreover, our analysis indicated a serious problem that the institution members are not familiar with currently available guidelines. It is an urgent task to disseminate the above described guidelines available at present. Taken together, our association with the help of guidelines needs to disseminate basic knowledge such as the infection rate, and the strategies for infection control in HIV, and provide the appropriate environment for imagery rehearsal through educational lectures, a symposium or lectures by the facilities that have accepted HIV-positive patients.

Table 8 Knowledge of HIV related guidelines

The Guidelines for Dialysis of HIV Positive Patients' published by Japanese Association of Dialysis Physicians and Japanese Society for Dialysis Therapy in 2010

$\begin{array}{llll}\text { Very familiar } & \text { Aware without practice } & \text { Vaguely familiar } & \text { Unknown } \\ 11.3 \% & 43.2 \% & 38.6 \% & 7.4 \%\end{array}$

Number of valid responses 2552

(some facilities selected several answers at the same time)

The Guidelines on Fundamental Handling and Infection Control in Dialysis Facilities in the fourth revised edition' published in 2015

$\begin{array}{llll}\text { Very familiar } & \text { Aware without practice } & \text { Vaguely familiar } & \text { Unknown } \\ 20.2 \% & 46.7 \% & 26.7 \% & 6.7 \%\end{array}$

Number of valid responses 2553

(some facilities selected several answers at the same time)

'Treatment Guidelines for HIV' published by research division of Ministry of Health, Labour and Welfare in 2016

$$
\begin{array}{llll}
\text { Very familiar } & \text { Aware without practice } & \text { Vaguely familiar } & \text { Unknown } \\
6.1 \% & 27.7 \% & 42.8 \% & 23.6 \%
\end{array}
$$

Number of valid responses 2546
The intention to accept HIV positive patients in the future

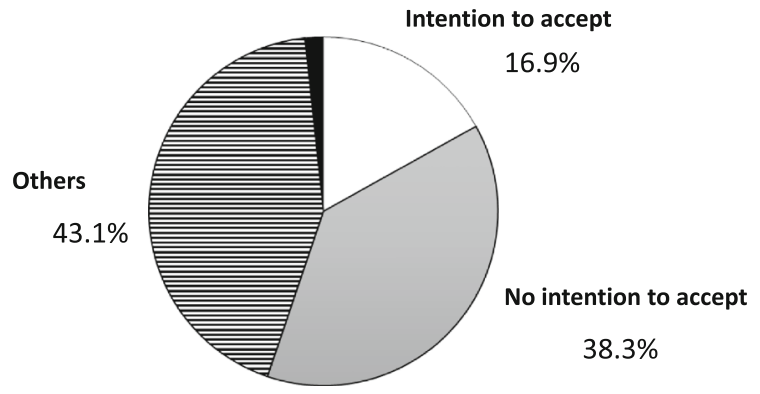

The number of valid responses

2,538

Fig. 3 The intention to accept HIV-positive patients in the future. Two thousand five hundred thirty-eight facilities responded to this question. While only $16.9 \%$ of the facilities intended to accept HIVpositive patients, $38.3 \%$ did not. The proportion of facilities that selected "others" as a response accounted for $43.1 \%$, which might suggest their unwillingness to accept HIV-positive patients or their challenges in accepting these patients

Furthermore, our association could set up a support section for the acceptance of HIV patients requiring dialysis.

Our questionnaire survey highlighted that many dialysis facilities did not have a system for accepting HIV-positive patients including a manual for needle piercing accidents, stocks of anti-HIV drugs, or develop cooperation with HIV core hospitals. While the manual for a needle piercing accident was prepared at more than $95 \%$ of the facilities that accept HIV patients, the figure

The opinions of facilities on informing the results of this questionnaire survey to HIV core hospitals

facilities that intended to accept

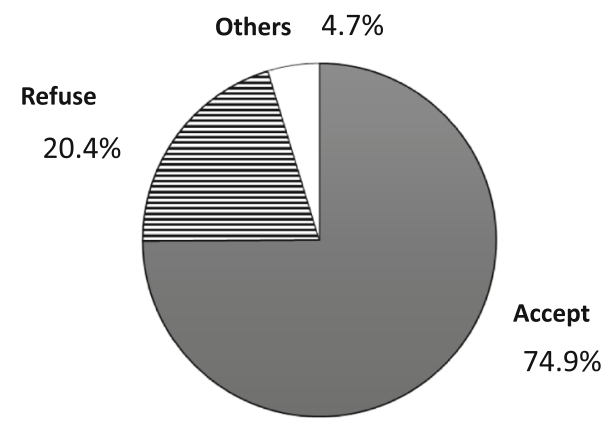

Number of valid responses 426

Fig. 4 The opinions of facilities on informing the results of this questionnaire survey to HIV core hospitals. The opinion to inform the results of this questionnaire survey to HIV core hospitals was evaluated in 426 facilities that intended to accept HIV-positive patients; $74.9 \%$ of the facilities accepted and $20.4 \%$ refused to inform 
was not $100 \%$ (Table 4). It is desirable to establish a cooperative relationship between the dialysis facilities and HIV core hospitals to accept HIV patients, allowing the latter to help the former by providing useful information in an emergent situation. Another reason why dialysis facilities may not be willing to accept HIV patients is the economic burden of stocking anti-HIV drugs for needle piercing accidents. In fact, $51.1 \%$ of the facilities stock anti-HIV drugs and $78.8 \%$ of these pay for it themselves. The currently recommended preventative medicine for a needle piercing accident is a combination of twice a day Raltegravir (Isentress ${ }^{\circ}$ ) and once a day Truvada (Truvada Combination Tablet). In an emergency situation, it is recommended that the person with exposure takes each tablet with their own consent and visit for consultation to a HIV core hospital. However, they generally leave the cost for preventative therapy to the dialysis facilities. This is not preferable for the dialysis facilities because these medicines are expensive (1510.4 yen/tablet for Raltegravir and 3756.3 yen/tablet for Truvada) and have a limited expiration date (30 months for Raltegravir and 3 years for Truvada). However, this problem could be solved by sharing of anti-HIV drug stocks through a collaborative group purchase by the HIV core hospitals. Thus, constructive discussions between the Japanese Society for AIDS Research and Japanese Society for Dialysis Therapy are needed to build an effective bounty system to reduce the economic burden on dialysis facilities.

The third problem is the low cooperation rate $(8.9 \%)$ with HIV core hospitals among all concerned facilities (Table 7). "Treatment Guidelines for HIV" published by the Research Division of Ministry of Health, Labour and Welfare describes that HIV core hospitals need to cooperate with medical facilities in advance [6]. Though some facilities refused to transfer the results of this questionnaire survey to the HIV core hospitals, it may be useful to generate a list of dialysis facilities that have already accepted the transfer and distribute it to the HIV core hospitals. Moreover, a collaborative meeting between the facilities and HIV core hospitals to accept patients, led by our association could help to expand the acceptance rate by exchanging information not only between dialysis facilities and HIV core hospitals, but also among the dialysis facilities. The cooperation with HIV core hospitals is considered important as this would allow healthcare providers in the dialysis facilities to work in safer environments by access to updated information on preventative medicine and appropriate measures for a needle piercing accident from specialists at the HIV core hospitals. To solve these problems, we need to open the dialog with the Japanese Association for Infectious Diseases and the relevant academic societies. On the other hand, the fact that the frequency of facilities with intention to cooperate (17.9\%) was lower than that of facilities without intention to cooperate
(26.3\%) (Table 7), the fact that $45.0 \%$ of them pointed out the reason for refusal as lack of cooperation with HIV core hospitals (Table 6), and the fact that $20.4 \%$ of these refused to transfer the results of this questionnaire survey to HIV core hospitals (Fig. 4) suggest the difficulties behind the current approaches. Considering these points, member institutions may want to consider a more cautious approach rather than the traditional top-down approach through discussions and mutual understanding with the associated academic societies.

Though this questionnaire survey had a high response rate $(64.0 \%)$, suggesting a high interest in this theme by member institutions, $36.0 \%$ of these could be considered less interested or to have no plan to accept HIV-positive patients. These facilities might not only lack the experience practicing with HIV-positive patients but also the basic knowledge. It is quite important to proceed with the establishment of educational and medical systems to expand dialysis facilities that are willing to accept HIV patients by challenging the problems clarified through this questionnaire survey.

\section{Conclusion}

In Japan, improvements in the prognosis of HIV patients have led to an increase in the number of patients requiring maintenance dialysis. Our questionnaire survey clarifies the lack of sufficient preparation and social support system for accepting HIV-positive patients in dialysis centers. The spread of knowledge on HIV, the establishment of a support system, and the cooperation of HIV core hospitals are the urgent needs, which will undoubtedly lead the progress on increased acceptance of HIV-positive patients for dialysis.

\section{Acknowledgements}

We thank all the dialysis facilities for responding to the questionnaire survey.

Availability of data and materials

All data generated or analyzed during this study are included in this published article.

\section{Authors' contributions}

$A Y$ and MR analyzed the questionnaire survey and wrote the draft of this paper. YI, NO, YK, TS, YTakano, IT, KH, YN, NH, TY, SW, YTakemoto, and HN modified the draft. All authors read and approved the final manuscript.

Ethics approval and consent to participate Not applicable

Consent for publication

Not applicable

Competing interests

The authors declare that they have no competing interests.

\section{Publisher's Note}

Springer Nature remains neutral with regard to jurisdictional claims in published maps and institutional affiliations. 
Received: 17 June 2018 Accepted: 22 August 2018

Published online: 24 October 2018

\section{References}

1. The AIDS surveillance committee of Ministry of Health, Labour and Welfare in Japan. The annual report of AIDS surveillance 2017 [online]. http://api-net. jfap.or.jp/status/2016/16nenpo/16nenpo_menu.html. Accessed 6 May 2018.

2. Yanagisawa N, Ajisawa A, Ando M. Chronic kidney disease among HIV-infected indivisuals. J AIDS Res. 2013;15:63-70.

3. Yanagisawa N, Ajisawa A, Imamura A, Suganuma A, Tsuchiya K, Nitta K, Ando M. Prevalence of HIV infection among patients receiving chronic dialysis therapy in Japan. A nationwide study based on a questionnaire survey. J Jpn Soc Dial Ther. 2014;47:623-8. (In Japanese)

4. Working group of Japanese Association of Dialysis Physicians and Japanese Society for Dialysis Therapy for the dialysis for HIV positive patients HIV. The guideline for the dialysis of HIV positive patients 2010 (In Japanese).

5. Health Labour Sciences Research Grant; AIDS prevention Projects; Research group to overcome the problem of HIV infection and its complications Research group to proceed the dialysis for HIV positive patients. The guideline about fundamental handlings and the infection control in dialysis facilities in fourth revised edition 2015 (In Japanese).

6. Health Labour Sciences Research Grant; AIDS prevention Projects; Research group to overcome the problem of HIV infection and its complications. The treatment guidelines for HIV 2016 (In Japanese).

7. Yanagisawa N, Ando M, Ajisawa A, et al. Clinical characteristics of kidney disease in Japanese HIV-infected patients. Nephron Clin Pract. 2011;118(3):285-91.

8. Hara M, Yanagisawa N, Ohta A, et al. Increased non-HDL-C level linked with a rapid rate of renal function decline in HIV-infected patients. Clin Exp Nephrol. 2017;21(2):275-82

9. Rasch MG, Helleberg M, Feldt-Rasmussen B, et al. Increased risk of dialysis and end-stage renal disease among HIV patients in Denmark compared with the background population. Nephrol Dial Transplant. 2014;29(6):1232-8.

10. U.S. Public Health Service. Updated U.S. public health service guidelines for the management of occupational exposures to HBV, HCV, and HIV and recommendations for post-exposure prophylaxis. MMWR Recomm Rep. 2001;50(RR-11):1-52.

Ready to submit your research? Choose BMC and benefit from:

- fast, convenient online submission

- thorough peer review by experienced researchers in your field

- rapid publication on acceptance

- support for research data, including large and complex data types

- gold Open Access which fosters wider collaboration and increased citations

- maximum visibility for your research: over $100 \mathrm{M}$ website views per year

At $\mathrm{BMC}$, research is always in progress.

Learn more biomedcentral.com/submissions 